\title{
High expression of transforming acidic coiled coil-containing protein 3 strongly correlates with aggressive characteristics and poor prognosis of gastric cancer
}

\author{
MIAO YUN ${ }^{1-3^{*}}$, JIAN RONG $^{4 *}$, ZHI-RUI LIN ${ }^{2,3}$, YU-LONG HE $^{5}$, JIA-XING ZHANG $^{1}$, \\ ZHEN-WEI PENG ${ }^{1}$, LIN-QUAN TANG ${ }^{2,3,6}$, MU-SHENG ZENG ${ }^{2,3}$, QIAN ZHONG ${ }^{2,3}$ and SHENG YE ${ }^{1}$ \\ ${ }^{1}$ Department of Oncology, The First Affiliated Hospital, Sun Yat-sen University; ${ }^{2}$ State Key Laboratory of Oncology \\ in South China, Collaborative Innovation Center for Cancer Medicine, ${ }^{3}$ Department of Experimental Research, \\ Sun Yat-sen University Cancer Center; ${ }^{4}$ Department of Extracorporeal Circulation, ${ }^{5}$ Department of Gastrointestinal Surgery, \\ The First Affiliated Hospital, Sun Yat-sen University; ${ }^{6}$ Department of Nasopharyngeal Carcinoma, \\ Sun Yat-sen University Cancer Center, Guangzhou, Guangdong 510000, P.R. China
}

Received January 25, 2015; Accepted May 5, 2015

DOI: 10.3892/or.2015.4093

\begin{abstract}
Transforming acidic coiled coil-containing protein 3 (TACC3) is well understood to regulate mitotic spindle dynamics and centrosome integrity during mitosis. TACC 3 has been suggested to be deregulated in a variety of human malignancies and may be involved in the process of cancer progression. The aim of the present study was to determine the status of TACC 3 expression in gastric cancer (GC) and to clarify its clinical/prognostic significance. In the present study, we applied quantitative PCR (qPCR) and western blotting to examine TACC $3 \mathrm{mRNA}$ /protein expression in paired GC tissues and matched adjacent non-malignant tissues. Immunohistochemistry (IHC) was performed on a large cohort of 186 postoperative GC samples. Chi-square test, Kaplan-Meier analysis and Cox regression modelling were used to analyse the data. Upregulated mRNA and protein expression levels of TACC 3 were observed in the majority of the GC tissues based on qPCR and western blotting compared
\end{abstract}

Correspondence to: Professor Sheng Ye, Department of Oncology, The First Affiliated Hospital, Sun Yat-sen University, 58 Zhongshan 2nd Road, Guangzhou, Guangdong 510080, P.R. China

E-mail: yeshengsysu@163.com

Professor Qian Zhong, State Key Laboratory of Oncology in South China, Collaborative Innovation Center for Cancer Medicine, Department of Experimental Research, Sun Yat-sen University Cancer Center, No. 651, Dongfeng Road East, Guangzhou, Guangdong 510060 , P.R. China

E-mail: zhongqian@sysucc.org.cn

*Contributed equally

Key words: transforming acidic coiled coil-containing protein 3, gastric cancer, western blotting, quantitative PCR, immunohistochemistry, prognosis to the adjacent non-cancerous gastric tissues. Specific IHC staining for TACC 3 was predominantly identified in the cytoplasm of the cancer cells. A high expression of TACC 3 was detected in 102 of the $186(54.8 \%)$ tissue samples and was significantly associated with the extracapsular extension of the tumour $(\mathrm{P}<0.001)$, tumour relapse $(\mathrm{P}<0.001)$ and shortened overall survival in $\mathrm{GC}(\mathrm{P}<0.001)$. Further analysis demonstrated that the TACC 3 expression level stratified the patient outcome in stage II $(\mathrm{P}=0.040)$, stage III $(\mathrm{P}<0.001)$, $\mathrm{T} 3 / 4(\mathrm{P}<0.001), \mathrm{N}$ positive $(\mathrm{P}<0.001)$ and poorly differentiated/undifferentiated tumour subgroups $(\mathrm{P}<0.001)$. The Cox regression analysis suggested that a high expression of TACC3 was an independent prognostic factor for GC patients. The measurement of TACC 3 protein expression may be beneficial for predicting clinical outcomes for GC patients.

\section{Introduction}

Although the morbidity and mortality of gastric cancer (GC) have been on the decrease over the past few decades, GC remains a major public health issue worldwide. The prognosis of GC remains poor, with a 5 -year survival rate of $<30 \%$ (1). Approximately $70 \%$ of new cases occur in East Asia, including a higher rate of locoregionally advanced disease and a younger age at diagnosis (2). The standard of treatment for GC has been surgery and multimodal treatment (3). However, traditional methods used may not precisely predict the prognosis of GC patients after surgical excision. Thus, there is a need for novel biomarkers that distinguish between patients experiencing different clinical outcomes, facilitating the individualisation of treatments for GC patients.

Centrosomes are crucial microtubule organising centres in animal cells (4). The physiological function of centrosomes is responsible for organising the poles of the mitotic spindle and for equally segregating chromosomes into two daughter cells during mitosis. Previous findings have shown that centrosomal abnormalities may lead to aneuploidy and genomic instability, further contributing to neoplastic transformation and 
tumourigenesis $(5,6)$. Moreover, a previous study suggested that chromosomal aneuploidy is associated with the formation and progression of GC (7).

Members of the transforming acidic coiled-coil (TACC) family of proteins are centrosome- and microtubule-binding proteins that play an important role in the regulation of centrosome integrity, spindle stability and microtubule assembly during cell division (8-14). TACC3, the third member in the TACC family, contains a highly conserved C-terminal coiled coil domain that interacts with microtubules (15). TACC3 is expressed in certain human proliferative tissues, such as testis, bone marrow, lung, spleen, thymus and peripheral blood leukocytes (10). Regarding its function in mitosis, TACC3 was suggested to be involved in the process of tumourigenesis in various types of cancer through the regulation of the transcriptional activities and the proliferative properties of tumour cells (16). TACC3 was found to be upregulated in multiple malignancies, including ovarian and invasive breast cancer $(17,18)$. It has been suggested that a high expression of TACC3 is a prognostic indicator of poor clinical outcome in non-small cell lung cancer (NSCLC) (19). The elevated expression of TACC3 was associated with tumour metastasis and chemoresistance in cervical cancer (20). Moreover, TACC3 induced EGF-mediated epithelial mesenchymal transition (EMT) by activating the PI3K/Akt and ERK signalling pathways in cervical cancer $(21,22)$. Alternatively, the depletion of TACC3 induced tumour regression in vivo (23). From a gene mapping perspective, TACC3 is located on chromosome 4p16.3, which is vulnerable to genetic variation, leading to an increased risk for bladder cancer, multiple myeloma and ovarian adenocarcinoma (24-26). Furthermore, TACC3 is closely mapped to the fibroblast growth factor receptor 3 (FGFR3) gene, and this region is susceptible to forming an FGFR3-TACC3 fusion in a subset of glioblastoma multiforme (27). This fusion gene escapes microRNA regulation via 3'-UTR depletion and encodes an oncogenic protein that induces mitotic and chromosomal segregation defects, possibly leading to aneuploidy in glioblastoma (28). Furthermore, cells exhibiting high levels of TACC3 expression exhibited defective cell cycle checkpoints and repair systems, leading to genomic instability (29). These results indicated that TACC 3 may be an important factor that contributes to tumour development and progression, providing a novel potential target for various cancer therapies.

Although previous studies have investigated TACC3 expression in many human cancers, little is known regarding its role in GC. In the present study, we examined the expression pattern of TACC 3 in human GC samples. The correlation between TACC 3 expression and the clinicopathological characteristics and prognosis of GC patients was evaluated.

\section{Materials and methods}

Patients and tissue specimens. This retrospective study was conducted on a cohort of $186 \mathrm{GC}$ patients receiving surgical resection of the primary tumour at the First Affiliated Hospital of Sun Yat-sen University, Guangzhou, China, between January 2007 and May 2009. Subjects with R1/R2 margins, with distant metastasis and who died during the perioperative period were excluded from the present study. Age, gender, intraoperative examination, tumour location, WHO classification, histological differentiation, pathologic TNM (pTNM) staging classification of the stomach [according to the American Joint Committee on Cancer (AJCC), 7th edition, 2010], extracapsular extension and tumour emboli in the vasculature were assessed by reviewing the medical records, the pathological records and glass slides. The clinical data for these samples are described in detail in Table I. None of the patients received preoperative chemotherapy or radiotherapy. The patients with advanced disease received postoperative chemotherapy (5-FU alone, 5-FU and platinum or paclitaxel and platinum) with or without radiotherapy. Follow-up assessments were performed every 3 months postoperatively for the first 2 years, followed by every 6 months until the patient succumbed. The survival status of the patients was ascertained by April, 2014. Moreover, 30 paired GC and adjacent macroscopically normal gastric mucosal tissues were collected from the patients using the same criteria at the same hospital between October, 2013 and March, 2014. The tissue samples were rapidly frozen following collection and were stored in a $-80^{\circ} \mathrm{C}$ freezer until use for quantitative polymerase chain reaction (qPCR) and western blotting. Written informed consent was obtained from all the patients. The present study was approved by the Ethics Committee of Sun Yat-sen University, Guangzhou, China.

Western blotting. Eight pairs of GC tissues and matched adjacent non-malignant tissues were lysed in sample buffer containing complete protease inhibitors and PhosSTOP phosphatase inhibitor. The protein levels were quantified using a BCA protein assay kit (Pierce, Rockford, IL, USA). Then, $40 \mu \mathrm{g}$ of tissue protein lysates were mixed with loading buffer and heated at $95^{\circ} \mathrm{C}$ for $10 \mathrm{~min}$. The protein lysates were then separated via $7.5 \%$ SDS-polyacrylamide gel electrophoresis (PAGE) and transferred to an Immuno-Blot PVDF membrane (Pall Corp., Port Washington, NY, USA). The membrane was blocked with 5\% skim milk for $1 \mathrm{~h}$. Primary rabbit monoclonal antibodies against TACC3 (Abcam, Cambridge, MA, USA) and $\alpha$-tubulin (Proteintech Group, Chicago, IL, USA) were then applied at dilutions of 1:1,000 and 1:3,000, respectively.

RNA extraction and real-time fluorescent quantitative PCR. Total RNA from the surgically obtained paired tissues $(n=30)$ was extracted using TRIzol reagent (Invitrogen) according to the manufacturer's instructions. The RNA was pretreated with DNase, and 2- $\mu$ g aliquots were used for cDNA synthesis using random hexamers. The primers used to amplify TACC3 were: 5'-CCTCTTCAAGCGTTTTGAGAAAC-3' (sense) and 5'-GCCCTCCTGGGTGATCCTT-3' (antisense). $\beta$-actin was amplified as an internal control using the primers: 5'-CGCGAG AAGATGACCCAGAT-3' (sense) and 5'-GGGCATACCCCT CGTAGATG-3' (antisense). The amplification procedure was performed as follows: initial step at $95^{\circ} \mathrm{C}$ for $5 \mathrm{~min}$, followed by 40 cycles of $95^{\circ} \mathrm{C}$ for $15 \mathrm{sec}$ and $60^{\circ} \mathrm{C}$ for $30 \mathrm{sec}$. The instrumental software (SDS 2.0) was used to calculate the relative quantity of the amplified samples. Relative quantification was used to analyze TACC3 gene expression using the $2^{-\triangle \Delta C t}$ method.

Immunohistochemistry (IHC). IHC analysis was performed to examine the TACC3 expression levels in $186 \mathrm{GC}$ tissue specimens. The paraffin-embedded GC specimens were sliced into $4-\mu \mathrm{m}$ sections and placed in an oven at $65^{\circ} \mathrm{C}$ for 
Table I. Correlation of the TACC3 IHC score with the clinicopathological parameters.

\begin{tabular}{|c|c|c|c|c|}
\hline \multirow[b]{2}{*}{ Characteristics } & \multicolumn{3}{|c|}{ TACC 3 expression } & \multirow[b]{2}{*}{ P-value } \\
\hline & $\begin{array}{l}\text { All patients } \\
\text { N }(\%)\end{array}$ & $\begin{array}{c}\text { Low expression } \\
\mathrm{N}(\%)\end{array}$ & $\begin{array}{l}\text { High expression } \\
\text { N }(\%)\end{array}$ & \\
\hline Gender & & & & 0.281 \\
\hline Male & $112(60.2)$ & $47(42.0)$ & $65(58.0)$ & \\
\hline Female & $74(39.8)$ & $37(50.0)$ & $37(50.0)$ & \\
\hline Age (years) & & & & 0.055 \\
\hline$<65$ & $119(64.0)$ & $60(50.4)$ & $59(49.6)$ & \\
\hline$\geq 65$ & $67(36.0)$ & $24(35.8)$ & $43(64.2)$ & \\
\hline Tumour invasion (T) & & & & 0.327 \\
\hline $\mathrm{T} 1+\mathrm{T} 2$ & $51(27.4)$ & $26(51.0)$ & $25(49.0)$ & \\
\hline $\mathrm{T} 3+\mathrm{T} 4$ & $135(72.6)$ & $58(43.0)$ & $77(57.0)$ & \\
\hline Nodal invasion $(\mathrm{N})$ & & & & 0.063 \\
\hline Negative & 77 (41.4) & $41(53.2)$ & $36(46.8)$ & \\
\hline Positive & $109(58.6)$ & $43(39.4)$ & $66(60.6)$ & \\
\hline TNM staging, 7 th edition & & & & 0.179 \\
\hline 1 & $41(22.0)$ & $21(51.2)$ & $20(48.8)$ & \\
\hline 2 & $47(25.3)$ & $25(53.2)$ & $22(46.8)$ & \\
\hline 3 & $98(52.7)$ & $38(38.8)$ & $60(61.2)$ & \\
\hline Differentiation & & & & 0.900 \\
\hline Well/moderately & $54(29.0)$ & $24(44.4)$ & $30(55.6)$ & \\
\hline Poorly/undifferentiated & $132(71.0)$ & $60(45.5)$ & $72(54.5)$ & \\
\hline Extracapsular extension & & & & $<0.001^{\mathrm{a}}$ \\
\hline No & $99(53.2)$ & $60(60.6)$ & $39(39.4)$ & \\
\hline Yes & $87(46.8)$ & $24(27.6)$ & $63(72.4)$ & \\
\hline Tumour emboli in the vasculature & & & & 0.729 \\
\hline No & $169(90.9)$ & $77(45.6)$ & $92(54.4)$ & \\
\hline Yes & $17(9.1)$ & $7(41.2)$ & $10(58.8)$ & \\
\hline Relapse & & & & $<0.001^{\mathrm{a}}$ \\
\hline No & $108(58.1)$ & $61(56.5)$ & $47(43.5)$ & \\
\hline Yes & 78 (41.9) & $23(29.5)$ & $55(70.5)$ & \\
\hline
\end{tabular}

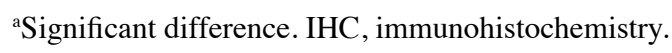

$2 \mathrm{~h}$. The sections were deparaffinised in xylene and rehydrated using an ethanol alcohol gradient followed by distilled water. Subsequently, the sections were immersed in $3 \%$ hydrogen peroxide for $15 \mathrm{~min}$ to quench endogenous peroxidase activity. The sections were boiled in citrate antigen retrieval solution ( $\mathrm{pH}$ 6.5) for $2.5 \mathrm{~min}$ in a high-pressure cooker for antigen retrieval. When the temperature of the antigen retrieval solution returned to room temperature, the sections were incubated in a primary antibody against TACC3 (1:800 dilution; Abcam, Cambridge, MA, USA) at $4^{\circ} \mathrm{C}$ overnight. As a negative control, the primary antibody was replaced with normal rabbit $\mathrm{IgG}$ at $4^{\circ} \mathrm{C}$ overnight while maintaining all the other treatment conditions. After washing three times for $5 \mathrm{~min}$ in $0.1 \%$ phosphate-buffered saline containing Tween-20 (PBST), the tissue sections were treated with a secondary antibody at $37^{\circ} \mathrm{C}$ for $30 \mathrm{~min}$. After three further washes in PBST, the tissue sections were stained with 3,3-diaminobenzidine (DAB) for $10 \mathrm{~min}$, counterstained with $10 \%$ Mayer's haematoxylin, dehydrated and mounted. Negative controls were performed concurrently by replacing the primary antibody with PBST. Positive control tissue was used as per the manufacturer's recommendation.

IHC analysis. The degree of immunostaining of the sections was scored by two independent pathologists who were blinded to the clinical parameters. For TACC3, only cytoplasmic staining was scored, while negligible nuclear or membrane staining was observed. Cytoplasmic immunoreactivity was scored in a semi-quantitative manner by calculating the ratio of positively stained tumour cells to the total tumour cells in $5 \%$ increments at a range of $0-100$ (P0, P1-3). A combined score was derived from the product of the percentage of positively stained tumour cells and the staining intensity. The staining intensity was classified as 0 (no staining), 1+ (weak), $2+$ (moderate) or $3+$ (strong) $(\mathrm{I} 0, \mathrm{I} 1-3)$. Each IHC score was 
a

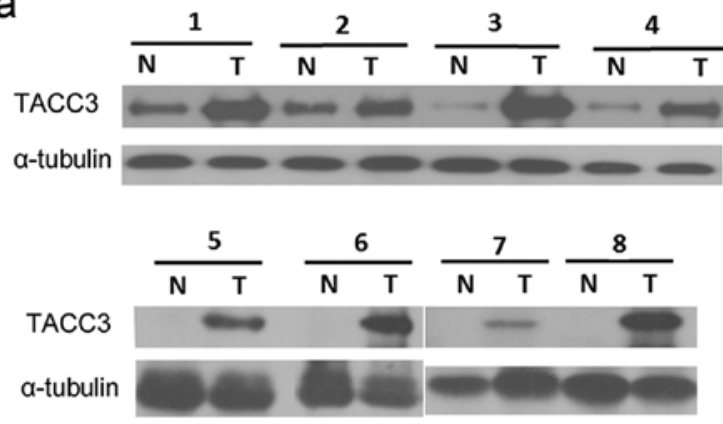

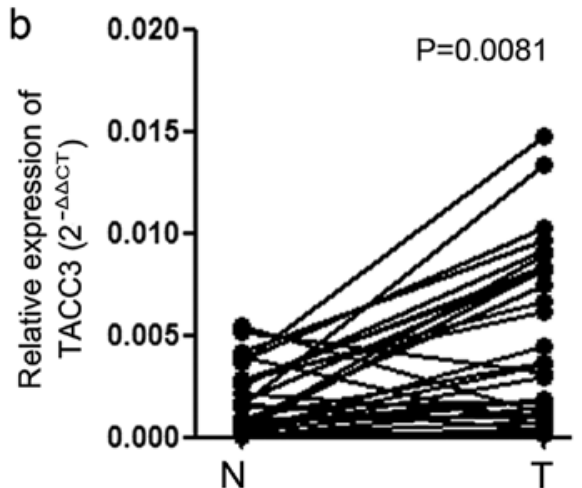

Figure 1. The protein and mRNA expression of TACC3 in GC and adjacent non-malignant gastric tissues. (a) Upregulated expression of TACC3 was detected via western blotting in $8 \mathrm{GC}$ tissues compared with the matched non-cancerous gastric tissues. The expression levels were normalised to those of $\alpha$-tubulin. (b) The mRNA expression of TACC 3 was significantly elevated in 30 pairs of GC tissues based on qPCR. The expression levels were normalised to those of $\beta$-actin. N, adjacent normal gastric tissue; T, GC tissue.

calculated based on the staining intensity and percentage scores according to the equation: $\mathrm{IHC}$ score $=\mathrm{I} 1 \mathrm{x} \mathrm{P} 1+\mathrm{I} 2 \mathrm{x}$ $\mathrm{P} 2+\mathrm{I} 3 \times \mathrm{P} 3$ (ranging from 0 to 300 ).

Selection of the threshold IHC score for a high TACC 3 expression. The threshold IHC score for a high expression of TACC 3 was determined using the receiver operating characteristic (ROC) curve analysis. A final IHC score for each tumour was derived by averaging the replicated scores. The sensitivity and specificity for discriminating between death and survival in this study was plotted, followed by the generation of a ROC curve. The IHC score that was closest to the point of maximal sensitivity and specificity was selected as the threshold value (30).

Statistical analysis. Statistical analyses were performed using SPSS v19.0 (IBM) and Prism software (GraphPad). $\mathrm{P}<0.05$ was considered to be significant. The Chi-square test or Fisher's exact test was used to evaluate the correlation between TACC 3 expression and the clinicopathological variables. Disease-free survival (DFS) was defined as the time from the initial treatment to cancer progression, metastasis, or death. Overall survival (OS) was defined as the time from the initial treatment to death. Estimates of the cumulative survival distributions were calculated using the Kaplan-Meier method, and differences between the groups were compared using the log-rank test. The significance of various clinicopathological characteristics was assessed using a univariate Cox regression model and multivariate Cox proportional hazard regression analysis followed by the log-rank test. The variables that were found to be significant based on the univariate analysis were selected for inclusion in the multivariate Cox model.

\section{Results}

Clinicopathological characteristics of the GC patients. The clinicopathological characteristics of the $186 \mathrm{GC}$ patients in this study are shown in Table I. The median follow-up duration was 62.3 (range, 1.2-87.2) months. The patients underwent total or subtotal gastrectomy with or without lymph node dissection, depending on the tumour location. A total of 112 males and 74 females were included in the present study, with a mean age of $59.2 \pm 12.5$ years. At the time of surgery, the percentage of patients classified as T1, T2, T3 and T4 was $16.7,10.8,2.2$ and $70.3 \%$, respectively. A total of $109(58.6 \%)$ patients exhibited lymph node involvement. The percentage of patients with stage I, II and III disease was 22.0, 25.3 and $52.7 \%$, respectively. Any patients exhibiting distant metastases were not included in the present study. As for the pathological type, 159 (85.5\%) patients were diagnosed with adenocarcinoma, and other pathological types included signet ring cell carcinoma (4.8\%), tubular adenocarcinoma (3.8\%), mucinous adenocarcinoma (3.2\%), papillary carcinoma (1.1\%), neuroendocrine carcinoma (1.1\%), and undifferentiated carcinoma $(0.5 \%)$. Regarding the tumour location, $52(28.0 \%)$ cases were located in the esophagogastric junction or the fundus of the stomach, 39 (21.0\%) cases were located in the middle of the stomach, $82(44.1 \%)$ cases were located in the lower portion of the stomach, $1(0.5 \%)$ case exhibited a diffuse lesion, and $12(6.5 \%)$ cases exhibited a tumour in an unspecified location.

Expression level of TACC3 based on western blotting and $q P C R$. We used western blotting to examine the expression of TACC 3 in 8 paired GC and adjacent non-cancerous tissues. The paired samples exhibited a higher TACC 3 expression in the tumour tissues than in the adjacent non-cancerous tissues (Fig. 1a). We also employed qPCR to detect the TACC 3 mRNA expression level in 30 paired tissues. The results of qPCR revealed a consistent trend of higher mRNA expression of TACC 3 in the GC tissues ( $\mathrm{P}=0.0081)$ (Fig. 1b).

IHC analysis of TACC 3 in clinical GC cases. Based on further investigation of the TACC 3 expression level in GC tissues via IHC, TACC 3 immunoreactivity was predominantly found in the cytoplasm of carcinoma cells and was also detected in specific areas of intestinal metaplasia tissue and in certain highly proliferative lymphocytes in the form of clusters of yellow-brown granules (Fig. 2b). Weak or negative TACC3 staining was observed in adjacent normal gastric epithelial cells (Fig. 2a) and in surrounding mesenchymal cells. The four categories of the intensity of TACC 3 immunostaining are shown in Fig. 3. According to ROC analysis, the patients were separated into two groups: low TACC 3 expression (IHC score $\leq 50)$ and high TACC 3 expression (>50). TACC 3 expression 


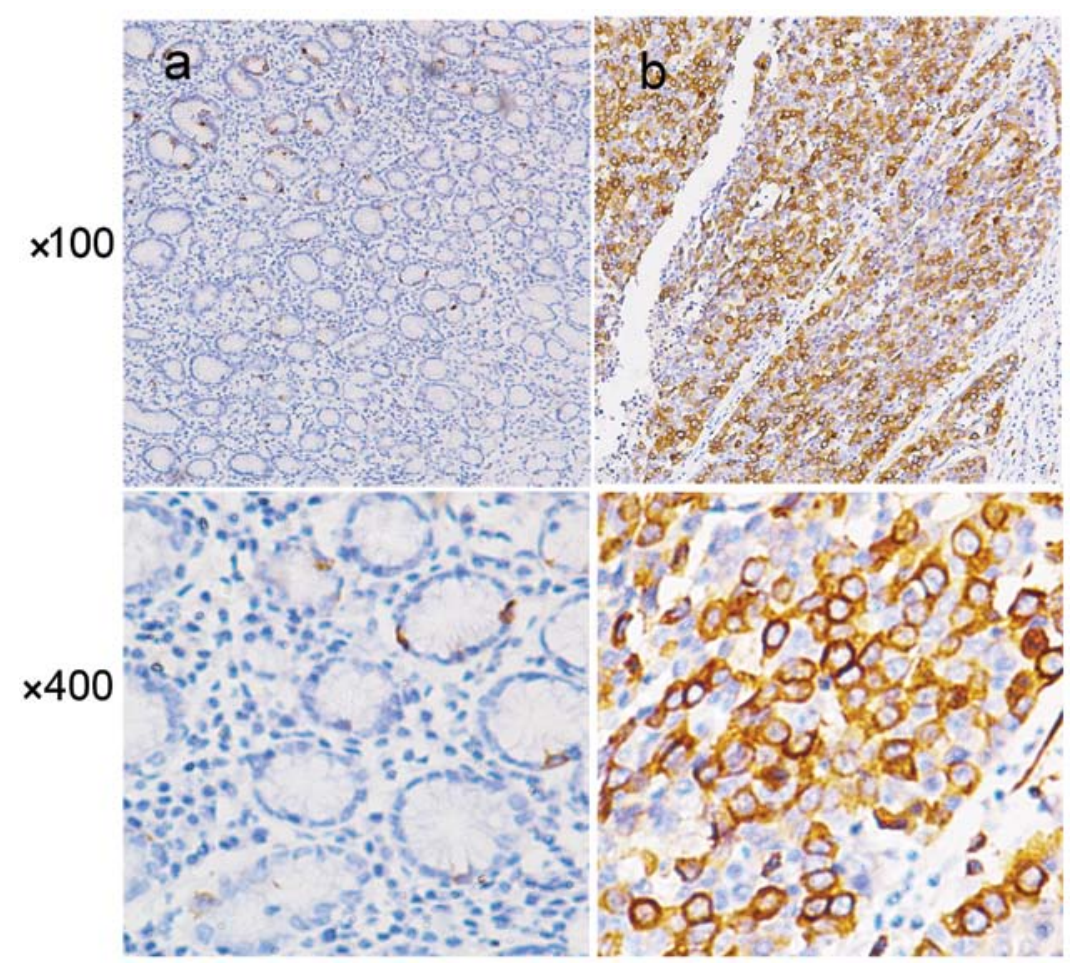

Figure 2. Representative images of TACC 3 expression in normal gastric epithelial and GC tissues via IHC. (a and b) TACC3 was absent from or only weakly detected in adjacent normal gastric epithelial cells, whereas it was positively detected in GC cells (original magnification, x100 and x400).

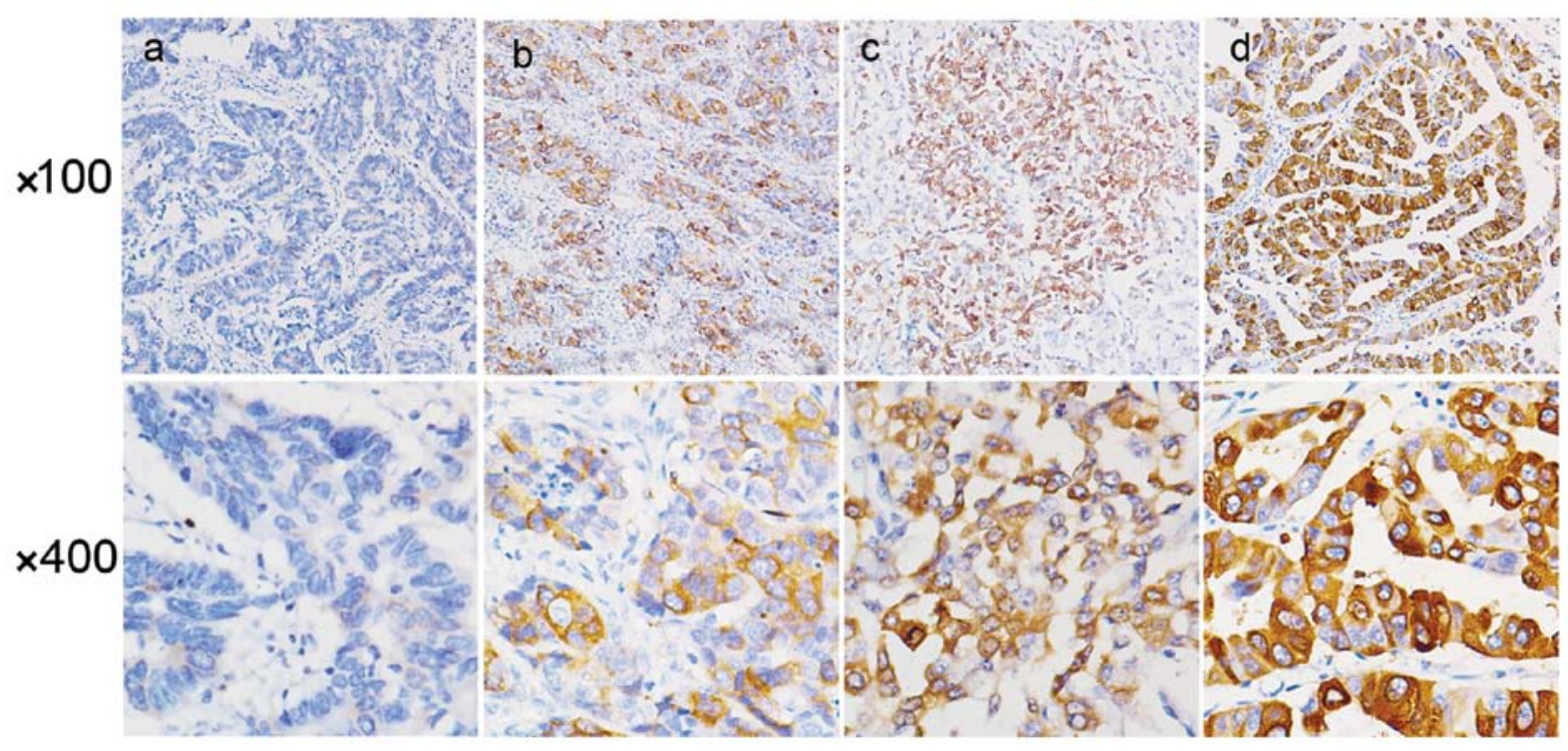

Figure 3. The staining intensity for TACC3 differed between specimens. The representative images show different staining intensities of TACC3: (a) negative staining, (b) weak staining, (c) moderate staining, and (d) strong staining (original magnification, $\mathrm{x} 100$ and x400).

(AUC $=0.672, \mathrm{P}<0.001)$ demonstrated a significant association with the survival status. Furthermore, 102 out of $186(54.8 \%)$ cases exhibited a high expression of TACC 3 in the tissue.

TACC3 expression and its association with the clinicopathological characteristics. We analysed the association between TACC3 protein expression and the clinicopathological characteristics using the Chi-square test or Fisher's exact test. This analysis revealed that a high expression of TACC 3 positively correlated with extracapsular extension $(\mathrm{P}<0.001)$ and tumour relapse $(\mathrm{P}<0.001)$. However, we failed to detect a correlation between TACC3 expression and other standard clinicopathological variables, including age, gender, pathological $\mathrm{T}$ stage, $\mathrm{N}$ stage, TNM staging status, tumour grade and the status of tumour emboli in the vasculature $(\mathrm{P}>0.05)$ (Table I).

High TACC3 expression based on IHC is associated with reduced survival in postoperative GC. A total of 78 deaths 

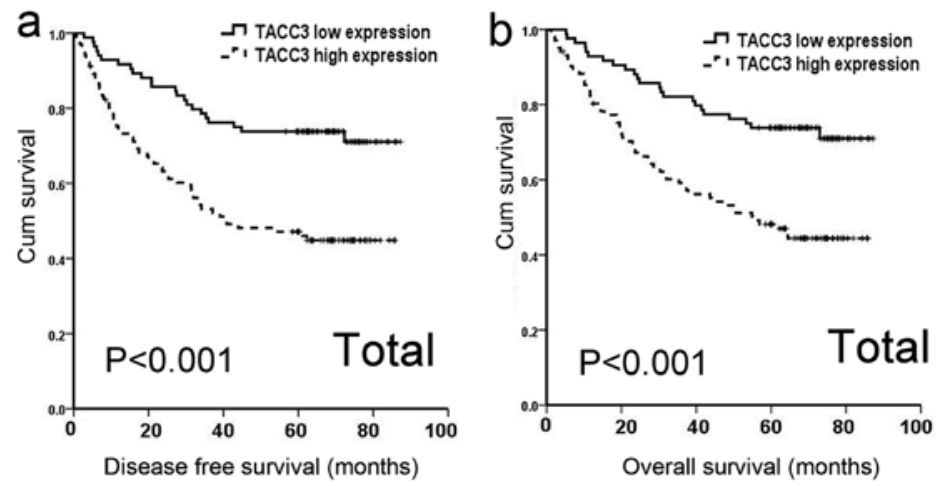

Figure 4. Survival curves for 186 GC patients according to TACC3 expression (log-rank test). (a) Probability of DFS for all GC patients: low TACC3 expression, $\mathrm{n}=84$; high TACC 3 expression, $\mathrm{n}=102$. (b) Probability of OS for all GC patients: low TACC 3 expression, $\mathrm{n}=84$; high TACC 3 expression, $\mathrm{n}=102$.

a

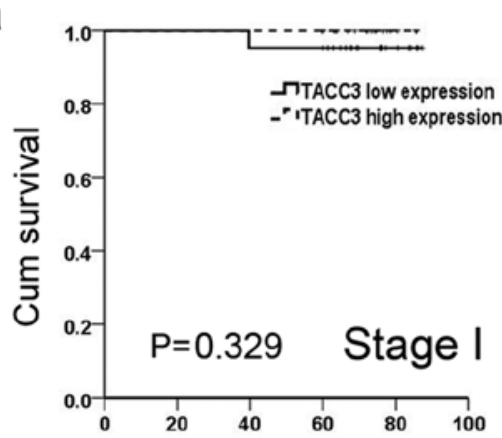

Overall survival (months)

d

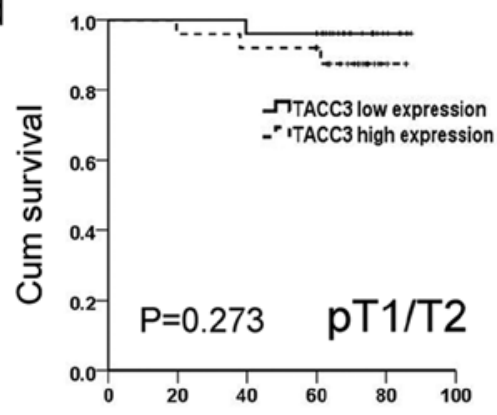

Overall survival (months)

g

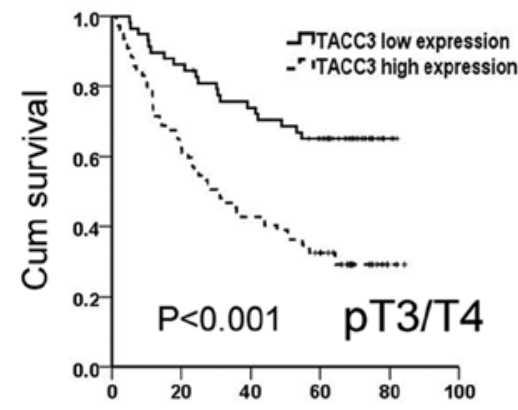

Overall survival (months) b

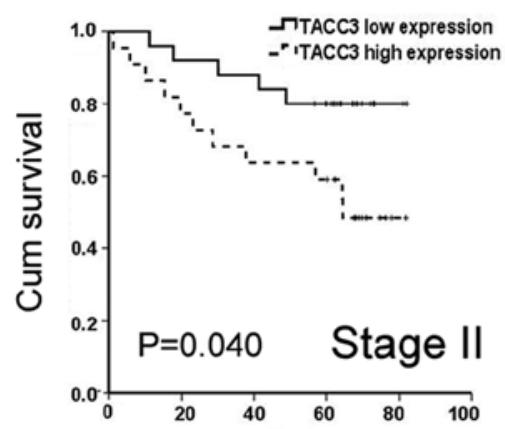

Overall survival (months)

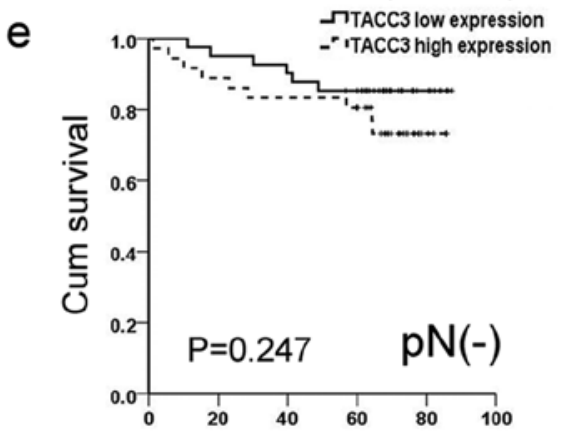

Overall survival (months)

h

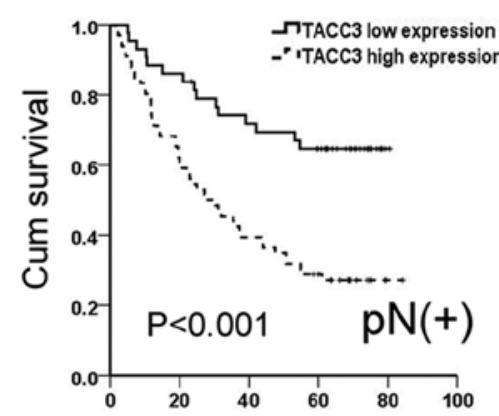

Overall survival (months)
C

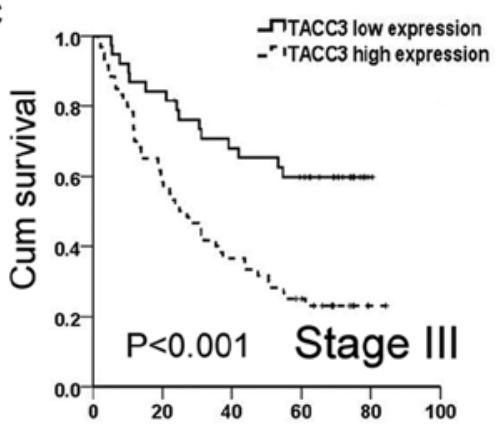

Overall survival (months)

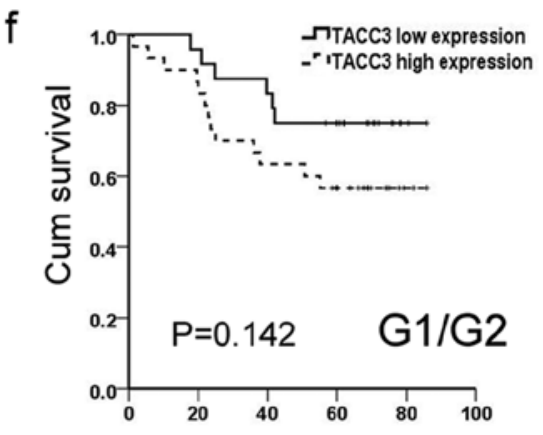

Overall survival (months)

i

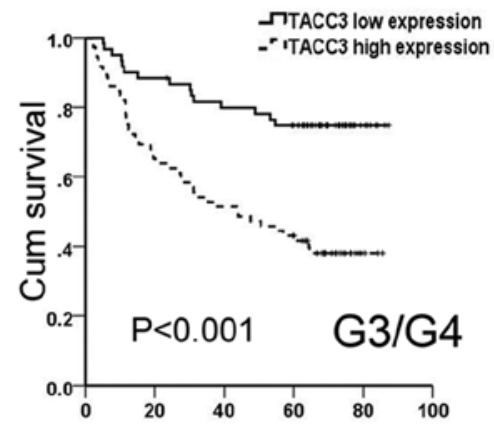

Overall survival (months)

Figure 5. Survival analysis according to TACC 3 expression in subsets of GC patients with different stages, pT/pN status and histological grade (log-rank test). Probability of OS for: (a) the stage I GC subgroup: low TACC 3 expression, n=21; high TACC 3 expression, n=20; (b) the stage II GC subgroup: low TACC 3 expression, $\mathrm{n}=25$; high TACC 3 expression, $\mathrm{n}=22$; (c) the stage III GC subgroup, low TACC 3 expression, $\mathrm{n}=38$; high TACC 3 expression, $\mathrm{n}=60$; (d) the pT 1 and pT2 GC subgroup, low TACC 3 expression, $n=26$; high TACC 3 expression, $n=25$; (e) the $\mathrm{pN}(-)$ GC subgroup, low TACC 3 expression, $n=41$; high TACC 3 expression, $\mathrm{n}=36$; (f) the G1/G2 GC subgroup, low TACC 3 expression, $\mathrm{n}=24$; high TACC 3 expression, $\mathrm{n}=30$; (g) the pT 3 and pT4 GC subgroup, low TACC3 expression, $\mathrm{n}=58$; high TACC 3 expression, $\mathrm{n}=77$; (h) the $\mathrm{pN}(+)$ GC subgroup: low TACC3 expression, $\mathrm{n}=43$; high TACC 3 expression, $\mathrm{n}=66$; and (I) the G3/G4 GC subgroup, low TACC 3 expression, $\mathrm{n}=60$; high TACC 3 expression, $\mathrm{n}=72$. 
(41.9\%) had occurred at the time of our analysis, with a median follow-up duration of 62.1 (range, 1.2-87.2) months for the surviving patients. The patients exhibiting a high TACC 3 expression exhibited a significantly shorter DFS (49.1 vs. 69.6 months, log-rank test $\mathrm{P}<0.001)$ and OS (50.9 vs. 72.0 months, log-rank test $\mathrm{P}<0.001)$ than the patients exhibiting a low expression of TACC3 (Fig. 4). Moreover, survival analysis was performed according to the level of TACC 3 expression in subsets of GC patients at different clinical stages, $\mathrm{pT}$ statuses, $\mathrm{pN}$ statuses and histological grades. Our results demonstrated that a high expression of TACC 3 was a prognostic factor for the stage II $(\mathrm{P}=0.040$, Fig. 5b), stage III ( $\mathrm{P}<0.001$, Fig. 5c), pT3/T4 ( $\mathrm{P}<0.001$, Fig. 5g), pN-positive $(\mathrm{P}<0.001$, Fig. 5h), and poorly differentiated/undifferentiated tumour subgroups of GC patients $(\mathrm{P}<0.001$, Fig. $5 \mathrm{i})$. However, the level of TACC 3 expression did not differentiate the outcomes of stage I ( $\mathrm{P}=0.329$, Fig. 5a), pT1/T2 ( $\mathrm{P}=0.273$, Fig. 5d), pN0 ( $\mathrm{P}=0.247$, Fig. 5e) or well- to moderately differentiated tumour subgroups ( $\mathrm{P}=0.142$, Fig. $5 f)$.

Univariate and multivariate analysis of the prognostic factors. The prognostic value of TACC 3 expression was confirmed by Cox regression analysis. Clinical characteristics, including age, gender, histological grade, tumour emboli in the vasculature, $\mathrm{T}$ status, $\mathrm{N}$ status, extracapsular extension, and TACC 3 expression were included in the univariate analysis to assess their impact on the survival of GC patients. Based on this analysis, T status, $\mathrm{N}$ status, extracapsular extension, and TACC 3 expression were found to impact survival. Therefore, these factors were further examined using a multivariate analysis model. Ultimately, T status, $\mathrm{N}$ status, and TACC 3 expression were identified as independent prognostic factors (Table II). Thus, the results indicated that the TACC 3 expression level significantly correlates with the prognosis of GC.

\section{Discussion}

The present study has provided, to the best of our knowledge, the first evidence that high TACC 3 expression correlates with shorter survival, tumour recurrence and extracapsular extension in GC patients. In the present study, we first performed western blotting and qPCR to examine the expression level of TACC 3 in several paired GC and adjacent non-neoplastic tissues. TACC 3 was upregulated at the protein and mRNA levels in the tumour tissues compared with the normal tissues. We then evaluated the protein expression of TACC 3 using IHC in a cohort of $186 \mathrm{GC}$ cases for whom follow-up data were available. We observed that the TACC 3 protein was predominantly detected in the cytoplasm of gastric malignancies and was increased in $54.8 \%$ of the GC tissue samples. By contrast, the normal gastric mucosal tissue exhibited an absent or low expression of TACC 3 . A further correlation analysis revealed that a high level of TACC 3 protein expression significantly correlated with extracapsular extension and tumour recurrence. Moreover, the patients with a high TACC 3 expression exhibited shortened OS and DFS. Taken together, these findings suggest that TACC 3 is associated with GC progression and is a potential independent prognostic indicator of survival for GC patients.

TACC 3 is a microtubule- and centrosome-associated protein that regulates mitotic spindle dynamics and centrosome integrity 
during mitosis. Accumulating evidence suggests that alterations of TACC3 expression occur in different cancer tissues. We observed an elevated expression of TACC 3 in GC and intestinal metaplasia tissues compared to adjacent non-cancerous gastric mucosal tissue, which was consistent with the data obtained using the database Oncomine $(16,31)$. Similar results were obtained from other studies that revealed that the TACC3 protein was upregulated in non-small cell lung cancer, multiple myeloma and cervical squamous cell carcinoma $(19,21,32)$. Moreover, Ma et al (18) showed that the levels of TACC3 mRNA were increased during the transition of breast cancer from ductal carcinoma in situ (DCIS) to invasive ductal carcinoma (IDC), suggesting that TACC3 expression corresponds to the progression status of the tumour and may facilitate the invasive phenotype of human malignancy. However, the downregulation of TACC 3 expression was detected in thyroid and ovarian papillary serous carcinoma $(33,34)$. These discrepancies in TACC3 expression between these studies may be due to its different roles in various types of cancer. The up- and downregulation of TACC3 may contribute to tumourigenesis by altering key cell processes, activating oncogenic signal transduction pathways and inducing genomic instability (16). Different molecular signal pathways may mediate its abnormal expression. Further analysis is required to elucidate the precise contribution of TACC 3 to GC and other cancer types, as well as the underlying mechanisms involved.

In the present study, a high expression of TACC 3 significantly correlated with an aggressive property of GC, extracapsular invasion, although no significant difference in TACC3 expression was found between different stages of GC. The patients with a high TACC3 expression more frequently experienced tumour recurrence than those exhibiting a low TACC 3 expression. Previous studies demonstrated that TACC3 is involved in the regulation of cell growth/differentiation and gene transcription $(35,36)$. Cells expressing high levels of TACC3 exhibited defective checkpoints and impaired DNA repair systems, leading to genomic instability (29). Moreover, elevated expression of TACC3 induces EGF-mediated epithelial-mesenchymal transition (EMT) by activating the PI3K/Akt and ERK signalling transduction pathways $(21,22)$. The alteration of TACC3 gene expression may confer the potential for the invasive growth of cancer cells. Taken together, these data may explain the reason for the high TACC3 expression positively correlating with an aggressive tumour property and with recurrence. Furthermore, a high expression of TACC3 was identified as an independent predictor of shorter OS in GC, as evidenced by the Kaplan-Meier curve and multivariate Cox regression analyses. Based on the subgroup analysis, TACC3 predicted the clinical outcome of the following subsets of GC patients: stage II, stage III, pT3/T4, lymph node metastasis and poorly differentiated/undifferentiated tumour. We found that a high expression of TACC3 is a significant factor associated with poor prognosis, especially in GC cases exhibiting aggressive characteristics. Patients with a high expression of TACC 3 should be more closely followed up and/or undergo more intensive treatment. Thus, TACC3 may be a potential marker of GC progression. Specifically, patients exhibiting a low TACC3 expression may opt for surgery and avoid unnecessary additional therapy, whereas those with a high TACC3 expression may benefit from high-dose adjuvant chemotherapy or targeted molecular therapy.
It is well known that the dynamic instability of microtubules is responsible for inaccurate chromosomal segregation during mitosis, and this process has been shown to be an effective target for cancer chemotherapy. Novel agents targeting non-microtubule mitotic proteins are currently under development as chemotherapeutic drugs (37). The study by Yao et al (23) demonstrated that depletion of TACC3 causes growth retardation of mouse thymic lymphoma and caused embryonic lethality to mice due to increased apoptosis, suggesting that TACC 3 may serve as a promising anticancer target. The induction of TACC 3 knockdown may accelerate the senescence of cancer cells in the presence of non-toxic paclitaxel concentrations. The combination of drugs targeting TACC 3 and drugs interfering with microtubule dynamics may efficiently improve the chemosensitivity of cancer cells (38). Yim et al (20) also reported that paclitaxel treatment and TACC3 knockdown using siRNA led to a significant synergistic enhancement of $\mathrm{G} 2 / \mathrm{M}$ phase arrest and apoptosis in HeLa cells. In addition, TACC 3 depletion sensitised cells to paclitaxel-induced death and superseded p21 WAF-mediated cell cycle arrest (39). From a therapeutic perspective, TACC3 may serve as a potential target for cancer therapy and as a synergistic target in combination with paclitaxel.

In summary, to the best of our knowledge, the present study has shown for the first time that TACC 3 expression was closely associated with extracapsular invasion of the tumour and tumour recurrence in GC. The expression of TACC3, as detected by IHC, may serve as a useful predictor of clinical outcome for GC patients. Thus, the expression level of TACC3 may be useful for assessing risk and optimising therapy.

\section{Acknowledgements}

This study was funded by the Key Grant of National Nature Science Foundation of China (grant no. 81230045). We would like to thank Dr Yuan Lin and Dr Yihong Ling for their contributions to the evaluation of the IHC scores.

\section{References}

1. Siegel R, Ma J, Zou Z and Jemal A: Cancer statistics, 2014. CA Cancer J Clin 64: 9-29, 2014.

2. Theuer CP, Kurosaki T, Ziogas A, Butler J and Anton-Culver H: Asian patients with gastric carcinoma in the United States exhibit unique clinical features and superior overall and cancer specific survival rates. Cancer 89: 1883-1892, 2000.

3. Sasako M, Inoue M, Lin JT, Khor C, Yang HK and Ohtsu A: Gastric Cancer Working Group report. Jpn J Clin Oncol 40 (Suppl 1): i28-i37, 2010.

4. Kellogg DR, Moritz M and Alberts BM: The centrosome and cellular organization. Annu Rev Biochem 63: 639-674, 1994.

5. Pihan GA and Doxsey SJ: The mitotic machinery as a source of genetic instability in cancer. Semin Cancer Biol 9: 289-302, 1999.

6. Sen S: Aneuploidy and cancer. Curr Opin Oncol 12: 82-88, 2000.

7. Sánchez-Pérez I, García Alonso P and Belda Iniesta C: Clinical impact of aneuploidy on gastric cancer patients. Clin Transl Oncol 11: 493-498, 2009.

8. Gómez-Baldó L, Schmidt S, Maxwell CA, Bonifaci N, Gabaldón T, Vidalain PO, Senapedis W, Kletke A, Rosing M, Barnekow A, et al: TACC3-TSC2 maintains nuclear envelope structure and controls cell division. Cell Cycle 9: 1143-1155, 2010.

9. Albee AJ and Wiese C: Xenopus TACC3/maskin is not required for microtubule stability but is required for anchoring microtubules at the centrosome. Mol Biol Cell 19: 3347-3356, 2008. 
10. Peset I and Vernos I: The TACC proteins: TACC-ling microtubule dynamics and centrosome function. Trends Cell Biol 18: 379-388, 2008

11. Yao R, Natsume Y and Noda T: TACC3 is required for the proper mitosis of sclerotome mesenchymal cells during formation of the axial skeleton. Cancer Sci 98: 555-562, 2007.

12. Schneider L, Essmann F, Kletke A, Rio P, Hanenberg H, Wetzel W, Schulze-Osthoff K, Nürnberg B and Piekorz RP: The transforming acidic coiled coil 3 protein is essential for spindledependent chromosome alignment and mitotic survival. J Biol Chem 282: 29273-29283, 2007.

13. Kinoshita K, Noetzel TL, Pelletier L, Mechtler K, Drechsel DN, Schwager A, Lee M, Raff JW and Hyman AA: Aurora A phosphorylation of TACC $3 /$ maskin is required for centrosomedependent microtubule assembly in mitosis. J Cell Biol 170: 1047-1055, 2005.

14. Gergely F, Draviam VM and Raff JW: The ch-TOG/XMAP215 protein is essential for spindle pole organization in human somatic cells. Genes Dev 17: 336-341, 2003.

15. Gergely F, Karlsson C, Still I, Cowell J, Kilmartin J and Raff JW: The TACC domain identifies a family of centrosomal proteins that can interact with microtubules. Proc Natl Acad Sci USA 97: 14352-14357, 2000.

16. Ha GH, Kim JL and Breuer EK: Transforming acidic coiled-coil proteins (TACCs) in human cancer. Cancer Lett 336: 24-33, 2013.

17. L'Espérance S, Popa I, Bachvarova M, Plante M, Patten N, Wu L, Têtu B and Bachvarov D: Gene expression profiling of paired ovarian tumors obtained prior to and following adjuvant chemotherapy: Molecular signatures of chemoresistant tumors. Int J Oncol 29: 5-24, 2006

18. Ma XJ, Salunga R, Tuggle JT, Gaudet J, Enright E, McQuary P, Payette T, Pistone M, Stecker K, Zhang BM, et al: Gene expression profiles of human breast cancer progression. Proc Natl Acad Sci USA 100: 5974-5979, 2003.

19. Jung CK, Jung JH, Park GS, Lee A, Kang CS and Lee KY: Expression of transforming acidic coiled-coil containing protein 3 is a novel independent prognostic marker in non-small cell lung cancer. Pathol Int 56: 503-509, 2006.

20. Yim EK, Tong SY,Ho EM, Bae JH, Um SJ and Park JS: Anticancer effects on TACC 3 by treatment of paclitaxel in HPV-18 positive cervical carcinoma cells. Oncol Rep 21: 549-557, 2009.

21. Ha GH, Kim JL and Breuer EK: TACC3 is essential for EGFmediated EMT in cervical cancer. PLoS One 8: e70353, 2013.

22. Ha GH, Park JS and Breuer EK: TACC3 promotes epithelialmesenchymal transition (EMT) through the activation of PI3K/ Akt and ERK signaling pathways. Cancer Lett 332: 63-73, 2013.

23. Yao R, Natsume Y, Saiki Y, Shioya H, Takeuchi K, Yamori T, Toki H, Aoki I, Saga T and Noda T: Disruption of Tacc3 function leads to in vivo tumor regression. Oncogene 31: 135-148, 2012.

24. Kiemeney LA, Sulem P, Besenbacher S, Vermeulen SH, Sigurdsson A, Thorleifsson G, Gudbjartsson DF, Stacey SN, Gudmundsson J, Zanon C, et al: A sequence variant at 4p16.3 confers susceptibility to urinary bladder cancer. Nat Genet 42 : 415-419, 2010

25. Seidl S, Kaufmann H and Drach J: New insights into the pathophysiology of multiple myeloma. Lancet Oncol 4: 557-564, 2003.

26. Birrer MJ, Johnson ME, Hao K, Wong KK, Park DC, Bell A, Welch WR, Berkowitz RS and Mok SC: Whole genome oligonucleotide-based array comparative genomic hybridization analysis identified fibroblast growth factor 1 as a prognostic marker for advanced-stage serous ovarian adenocarcinomas. J Clin Oncol 25: 2281-2287, 2007.
27. Singh D, Chan JM, Zoppoli P, Niola F, Sullivan R, Castano A, Liu EM, Reichel J, Porrati P, Pellegatta S, et al: Transforming fusions of FGFR and TACC genes in human glioblastoma. Science 337: 1231-1235, 2012.

28. Parker BC, Annala MJ, Cogdell DE, Granberg KJ, Sun Y, Ji P, Li X, Gumin J, Zheng H, Hu L, et al: The tumorigenic FGFR3TACC3 gene fusion escapes miR-99a regulation in glioblastoma. J Clin Invest 123: 855-865, 2013.

29. Ha GH, Kim JL, Petersson A, Oh S, Denning MF, Patel T and Breuer EK: TACC3 deregulates the DNA damage response and confers sensitivity to radiation and PARP inhibition. Oncogene 34: 1667-1678, 2015.

30. Zlobec I, Steele R, Terracciano L, Jass JR and Lugli A: Selecting immunohistochemical cut-off scores for novel biomarkers of progression and survival in colorectal cancer. J Clin Pathol 60: 1112-1116, 2007.

31. D'Errico M, De Rinaldis E, Blasi MF, Viti V, Falchetti M, Calcagnile A, Sera F, Saieva C, Ottini L, Palli D, et al: Genomewide expression profile of sporadic gastric cancers with microsatellite instability. Eur J Cancer 45: 461-469, 2009.

32. Still IH, Vince P and Cowell JK: The third member of the transforming acidic coiled coil-containing gene family, TACC 3 , maps in $4 \mathrm{p} 16$, close to translocation breakpoints in multiple myeloma, and is upregulated in various cancer cell lines. Genomics 58: $165-170,1999$.

33. Ulisse S, Baldini E, Toller M, Delcros JG, Guého A, Curcio F, De Antoni E, Giacomelli L, Ambesi-Impiombato FS, Bocchini S, et al: Transforming acidic coiled-coil 3 and Aurora-A interact in human thyrocytes and their expression is deregulated in thyroid cancer tissues. Endocr Relat Cancer 14: 827-837, 2007.

34. Lauffart B, Vaughan MM, Eddy R, Chervinsky D, DiCioccio RA, Black JD and Still IH: Aberrations of TACC1 and TACC 3 are associated with ovarian cancer. BMC Womens Health 5: 8, 2005.

35. Angrisano T, Lembo F, Pero R, Natale F, Fusco A, Avvedimento VE, Bruni CB and Chiariotti L: TACC3 mediates the association of MBD2 with histone acetyltransferases and relieves transcriptional repression of methylated promoters. Nucleic Acids Res 34: 364-372, 2006.

36. Sadek CM, Pelto-Huikko M, Tujague M, Steffensen KR, Wennerholm M and Gustafsson JA: TACC 3 expression is tightly regulated during early differentiation. Gene Expr Patterns 3: 203-211, 2003

37. Fielding AB, Lim S, Montgomery K, Dobreva I and Dedhar S: A critical role of integrin-linked kinase, ch-TOG and TACC3 in centrosome clustering in cancer cells. Oncogene 30: 521-534, 2011.

38. Schmidt S, Schneider L, Essmann F, Cirstea IC, Kuck F, Kletke A, Jänicke RU, Wiek C, Hanenberg H, Ahmadian MR, et al: The centrosomal protein TACC 3 controls paclitaxel sensitivity by modulating a premature senescence program. Oncogene 29: 6184-6192, 2010.

39. Schneider L, Essmann F, Kletke A, Rio P, Hanenberg H, Schulze-Osthoff K, Nürnberg B and Piekorz RP: TACC3 depletion sensitizes to paclitaxel-induced cell death and overrides p21WAF-mediated cell cycle arrest. Oncogene 27: 116-125, 2008. 